\title{
Availability of health facilities and utilization of maternal and newborn postnatal care in rural Malawi
}

\author{
Eunsoo Timothy Kim ${ }^{1,2^{*}}$, Kavita Singh ${ }^{1,2}$, llene S. Speizer ${ }^{1,3}$, Gustavo Angeles ${ }^{1,3}$ and William Weiss ${ }^{4}$
}

\begin{abstract}
Background: This study explored the role of health facility availability as it relates to maternal and newborn PNC use in rural Malawi.

Methods: Malawi Demographic and Health Survey (MDHS) 2015-16 data, MDHS 2015-16 household cluster GPS data, Malawi Service Provision Assessment (MSPA) 2013-14 data and MSPA 2013-14 facility GPS data were used. Household clusters were spatially linked with facilities using buffers. Descriptive analyses were performed and generalized estimating equations (GEE) were used to determine the effects of having different types of facilities at varying distances from household clusters on receipt of maternal and newborn PNC in rural Malawi.

Results: In rural Malawi, around 96\% of women had facilities providing PNC within $10 \mathrm{~km}$ of where they live. Among women who have clinic-level facilities within $5 \mathrm{~km}$ of where they live, around $25 \%$ had clinic-level facilities that provide PNC. For rural women who gave birth in the past 5 years preceding the survey, only about 3\% received maternal PNC within $24 \mathrm{~h}$ and about $16 \%$ received maternal PNC within the first week. As for newborn PNC, 3\% of newborns had PNC within $24 \mathrm{~h}$ and about 26\% had newborn PNC within the first week. PNC mostly took place at facilities (94\% for women and 95\% for newborns). For women who delivered at home, having a health center providing PNC within $5 \mathrm{~km}$ was positively associated with maternal and newborn PNC. For women who delivered at facilities, having a health center providing PNC within $5 \mathrm{~km}$ was positively associated with maternal PNC and having a health center providing PNC between $5 \mathrm{~km}$ and $10 \mathrm{~km}$ was positively associated with both maternal and newborn PNC. Regardless of the place of delivery and distance band, having a clinic-level facility providing PNC did not have significant positive effects on maternal and newborn PNC.
\end{abstract}

Conclusions: Providers should be trained to perform quality PNC at all facilities. It would also be important to address concerns related to health workers. Lastly, it would be key to increase community awareness about the importance of seeking timely PNC and about the utility of lower-level facilities for receiving preventative PNC.

Keywords: Postnatal care, Quality of care, Rural health, Maternal health, Newborn health, Malawi

\section{Background}

Two of the targets for Sustainable Development Goal (SDG) 3 are to universally reduce the maternal mortality ratio to less than 70 maternal deaths per 100,000 live births and neonatal mortality to at least as low as 12 neonatal deaths per 1000 live births by 2030 [1]. The

\footnotetext{
* Correspondence: timkim1207@gmail.com

'Department of Maternal and Child Health, Gillings School of Global Public Health, University of North Carolina at Chapel Hill, Chapel Hill, NC, USA ${ }^{2}$ MEASURE Evaluation/Carolina Population Center, University of North Carolina at Chapel Hill, Chapel Hill, NC, USA

Full list of author information is available at the end of the article
}

maternal mortality ratio in Malawi was 634 maternal deaths per 100,000 live births in 2015 [2]. This is higher than the sub-Saharan average of 546 maternal deaths per 100,000 live births in 2015 [3]. The neonatal mortality rate in Malawi was 22 deaths per 1000 live births in 2015 which was actually lower than the sub-Saharan average of 29 deaths per 1000 live births in 2015 [4] but still much higher than the SDG 3 target [1]. These statistics indicate that there is a need for further reduction in maternal and newborn mortality in Malawi and subSaharan Africa, more broadly. Malawi is one of the

(c) The Author(s). 2019 Open Access This article is distributed under the terms of the Creative Commons Attribution 4.0 International License (http://creativecommons.org/licenses/by/4.0/), which permits unrestricted use, distribution, and 
poorest countries in sub-Saharan Africa [5] and remains among the worst off on these key indicators despite the receipt of extensive amounts of overseas development funding [6]. In 2016, Malawi was the fifth largest recipient of overseas development assistance to the healthrelated sector [6]. The country received 718 million US dollars in 2016 from bilateral and multilateral donor organizations [6].

\section{Focus on postnatal care (PNC)}

The postnatal period, typically defined as the first 42 days after birth, is particularly a vulnerable time for both the mothers and their newborns because they are at a high risk of mortality during this period [7]. Common causes of maternal mortality in this period are postpartum hemorrhage, sepsis and infection [7]. For newborns, common causes of mortality include intrapartum related birth asphyxia, infection and prematurity among others [7]. Timely and proper PNC can offer a critical opportunity to potentially reduce preventable maternal and newborn deaths [7]. In terms of supporting evidence, however, no prior study has rigorously examined the association between receipt of maternal PNC and the reduction of maternal mortality [8]. Studies linking newborn PNC and the reduction of newborn mortality, particularly for facility deliveries, are also difficult to find. One study by Singh, Brodish and Haney examined the associations between newborn PNC by provider type and neonatal mortality in 10 sub-Saharan African countries [9]. This study found that PNC within the first week by both skilled and unskilled health providers were associated with reduction of newborn deaths between days 2 and 7 and also between days 2 and 28 [9]. Despite the apparent lack of high quality evidence, the WHO nevertheless strongly recommends PNC for both the mothers and the newborns to reduce morbidity and mortality [8].

During PNC, the WHO recommends that mothers are assessed for vaginal bleeding, uterine contraction, fundal height, temperature, heart rate and blood pressure within the first $24 \mathrm{~h}$ and continually monitored for danger signs afterwards [8]. For newborns, it is recommended that they are assessed for clinical danger signs such as poor feeding, convulsions, fast breathing, severe chest in-drawing, lack of spontaneous movement, very high or low body temperature and jaundice [8].

It is important that the recommended number, timing and content of maternal and newborn PNC services are provided equitably across the geographic and socioeconomic spectrums. According to a recent systematic review and meta-analysis of PNC services in low- and middle-income countries, women living in urban areas had significantly higher odds of using PNC services compared to women living in rural areas (OR: 1.36; 95\% CI: 1.01-1.81) [10]. This study concluded that within low- and middle-income settings, inequities exist in PNC use between rural and urban areas and also by education levels and socioeconomic status [10].

\section{The rural context}

In Malawi, over $80 \%$ of men and women live in rural areas [11]. Rural areas have different sociodemographic, geographic, and health service characteristics compared to their urban counterparts across the world [12]. Some of these differences pertain to natural geography, local climate, tradition, culture, poverty level, resource availability, road infrastructure and transportation availability [12]. When it comes to the provision of quality healthcare services, ensuring availability of transport, medicines, skilled health workforce and even health facilities is crucial for remote areas [12]. However, because such resources are scarce in rural areas, policy decision-makers need to consider prioritizing the most pressing needs of the communities under tight budgetary constraints [12].

One example of this predicament is whether to strengthen existing primary healthcare facilities or invest in the construction of new primary healthcare facilities to create greater availability in remote areas [13]. In either case, adequate funding support, community engagement, and health workforce competency and retention are integral elements in order for primary healthcare to gain trust in the communities even after the issue of availability is resolved [14]. This is especially true since the density of physicians, nurses and midwives in Malawi was merely 3 per 10,000 population in 2010 , which is critically lower than the World Health Organization (WHO) threshold of 23 physicians, nurses and midwives per 10,000 required to maintain essential levels of health services for mothers and children [15].

\section{Study aims}

Among the diversity of multiple competing rural health needs, this study examines the role of health facility availability as it relates to maternal and newborn PNC use in rural Malawi. Three questions are addressed: (1) What is the availability of health facilities providing PNC in rural Malawi? (2) Where and when do mothers and newborns receive PNC in rural Malawi? and (3) What are the effects of having different types of health facilities at varying distances from household clusters on receipt of maternal and newborn PNC in rural Malawi? These are important policy questions that are expected to contribute to health services research in the context of Malawi and sub-Saharan Africa at large.

\section{Methods}

Data sources

Several datasets from the Malawi Demographic and Health Survey (MDHS) program were used to create a 
master analysis dataset. First, woman's questionnaire data from the 2015-16 MDHS were retrieved [11]. The 2015$16 \mathrm{MDHS}$ was implemented using a two-stage cluster sampling design. In the first stage, all 28 administrative districts in Malawi were stratified into 56 urban and rural strata [11]. For each stratum, a sample of standard enumeration areas (SEA) was selected based on the complete list of SEAs derived from the 2008 sampling frame of the Malawi Population and Housing Census [11]. Selection of the SEAs also occurred in two stages [11]. One hundred seventy-three urban SEAs and 677 rural SEAs were independently selected using probability proportional to the size of the SEA [11]. Then, 30 households from urban clusters and 33 households from rural clusters were selected using an equal probability systematic selection from the complete list of households in selected SEAs [11]. Selected SEAs with more than 250 households were segmented due to their large size and only one segment of households with probability proportional to the segment size was used for household listing [11]. The woman's questionnaire collected various health and demographic data from all women in the reproductive age range between 15 and 49 years living in the selected households or who were found as visitors in the selected households on the day of the survey [11].

Second, the GPS coordinates of the centroids of the study clusters from the 2015-16 MDHS were linked with the woman's data through unique identifiers. The published GPS coordinates are not the exact locations of the study clusters because they have been systematically displaced using the "random direction, random distance" method [16]. This was done in order to protect the respondents from the threat of identity disclosure [16]. Each GPS coordinate was displaced a distance of up to two kilometers if it was an urban cluster. The majority of rural clusters (99\%) were displaced a distance of up to five kilometers [17]. A randomly selected $1 \%$ of the rural clusters were displaced a distance of up to ten kilometers [17].

The third data source was the 2013-14 Malawi Service Provision Assessment (MSPA). These data were collected from a census of public and private facilities in all 28 districts including facilities run by the government, Christian Health Association of Malawi, other faith-based organizations, non-governmental organizations, private for-profit organizations and others [18]. The 2013-14 MSPA includes a total of four different questionnaires - Facility Inventory, Health Provider Interview, Observation Protocols and Exit Interview questionnaires with select clients [18]. The information about whether health facilities provide PNC services was obtained from the health provider interview. At each facility, the goal was to interview an average of eight health providers. For facilities that had less than eight providers, every provider was interviewed. For larger facilities, providers who were deemed most knowledgeable about their facility were selected for interview. If any health provider mentioned that he or she provides PNC services, the corresponding health facility was labeled as one providing PNC services.

Then, the GPS coordinates of the health facilities in 2013-14 MSPA were spatially linked with the woman's questionnaire data from the 2015-16 MDHS. Three distance bands around household clusters were considered for the spatial linkage. Health facilities located between $0 \mathrm{~km}$ and $5 \mathrm{~km}(\leq 5 \mathrm{~km})$ were grouped as the closest distance band. Health facilities located between $5 \mathrm{~km}$ and $10 \mathrm{~km}(>5 \mathrm{~km}$ and $\leq 10 \mathrm{~km}$ ) were grouped as the midrange distance band. Health facilities located between $10 \mathrm{~km}$ and $15 \mathrm{~km}$ (>10 km and $\leq 15 \mathrm{~km}$ ) were grouped as the farthest distance band. Unlike the GPS coordinates of the 2015-16 MDHS household clusters, the GPS coordinates of the facilities were not displaced and reflect the true location [17]. Skiles, Burgert, Curtis and Spencer compared three data scenarios where methodological considerations of geographically linking DHS household clusters with health facilities were explored [19]. The study used the 2007 Rwanda Service Provision Assessment and the 2007-2008 Rwanda Interim Demographic and Health Survey [19]. In the study, the most ideal data scenario was having a census of all health facilities and undisplaced geographic locations of household clusters [19]. Other less ideal scenarios were either having a census of all health facilities and displaced household cluster locations or having a sample of health facilities and displaced household cluster locations [19]. The current study fits in with the second scenario where data were collected from a census of all health facilities in Malawi but the household cluster locations were randomly displaced. Skiles et al. reported that in the second scenario, using a Euclidean buffer of $5 \mathrm{~km}$ resulted in 5.9 to $9.2 \%$ of hospitals being misclassified, 7.0 to $12.4 \%$ of health centers being misclassified and 4.9 to $7.6 \%$ of health posts being misclassified [19]. The degree of misclassification error due to random displacement of household clusters in Malawi is expected to be similar to that reported in Skiles et al. but the possibility that there could be greater error in Malawian context cannot be ruled out completely.

The Woman's Questionnaire data from the 2015-16 MDHS, the GPS coordinates of the 2015-16 MDHS household clusters, the 2013-14 MSPA data and the GPS coordinates of the 2013-14 MSPA facilities are all publicly available on the Demographic and Health Surveys Program website [20] upon request.

\section{Variables}

All analyses were stratified by place of delivery. This is because types of health facilities and the proximity of 
these health facilities from household clusters are presumed to influence receipt of PNC differently based on where women delivered. Women who delivered at home may seek PNC at a health facility at the time of their choosing or receive a home visit by a health worker. In either circumstance, the proximity and the types of health facilities nearby women's homes can potentially influence their receipt of PNC. However, women who delivered at health facilities face a slightly different set of options. After delivery at the facility, women may receive PNC on site before returning home for the first time, return home first then seek PNC at a later time at a facility or return home first then receive a postnatal home visit by a health worker. Due to these differences in careseeking options based on place of delivery, there were several outcome variables used for analyses (in separate models). For women who delivered at home, the main outcomes were maternal PNC within 1 day of birth, newborn PNC within 1 day of birth, maternal PNC within 7 days of birth and newborn PNC within 7 days of birth. For women who delivered at health facilities, the main outcomes were maternal PNC between day 1 and day 7 and newborn PNC between day 1 and day 7 . PNC between day 1 and day 7 was considered because women who received PNC right after delivery but before leaving the facility (to return home for the first time) will most likely do so in the first $24 \mathrm{~h}$. Looking at this time interval can potentially capture the effects for women who were discharged and came back to a facility for a first or second postnatal check. As a supplementary analysis (see Additional file 1), PNC within the first day was still considered for women who delivered at health facilities to check for the assumption that some women receive PNC before discharge. In this case, the types of health facilities and their proximity should not have any significant positive influence on PNC seeking decisions because women are already at the facilities. All of the outcomes are binary with "1" indicating PNC in the specified time period and " 0 " otherwise. There were no women who responded "don't know" for maternal PNC. For newborn PNC, less than $1 \%$ of the women responded "don't know." Among all rural women who delivered in the 5 years prior to the survey, less than $1 \%$ of the women had missing data for maternal and newborn PNC.

There were three main types of binary indicators for health facilities: clinic-level facilities providing PNC, health centers providing PNC and hospitals providing PNC. Clinic-level facilities included maternities, dispensaries, clinics and health posts. Health centers only included facilities designated as health centers. Hospitals included central hospitals, district hospitals, rural/community hospitals and other hospitals. Health centers were set apart from other lower-level facilities because they comprise the largest number among all health facilities in Malawi [18]. In addition, compared to other lower-level facilities, health centers are much more likely to offer basic client services and delivery-related services in Malawi [18]. Types of facilities are meant to serve as indicators of the level of quality that can be provided at the facilities while three separate rings of buffers $(0-5 \mathrm{~km}$, $5-10 \mathrm{~km}$ and $10-15 \mathrm{~km}$ ) indicate different levels of proximity or distance from the household clusters. See Fig. 1 for a visual illustration.

Covariates in the models included season in which women gave birth, ownership of TV or a radio, whether cost of treatment is a perceived problem, women's age at the time of the survey, women's education, women's employment, household wealth, number of total births, newborn size, newborn sex, religion and region. For women who delivered at health facilities, cesarean section,

Presence of health facility located within $5 \mathrm{~km}$ of household cluster (providing PNC)

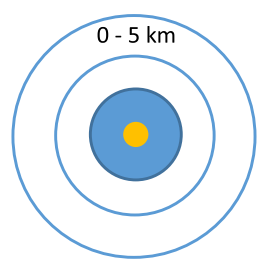

Clinic-level facilities

Health Centers

Hospitals

Presence of health facility located between $\mathbf{5} \mathrm{km}$ and $\mathbf{1 0}$ km from household cluster (provided PNC)

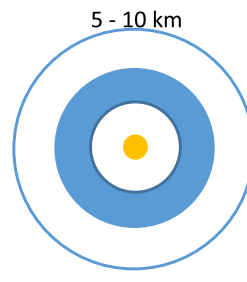

Clinic-level facilities

Health Centers

Hospitals

Presence of health facility located between $10 \mathrm{~km}$ and $15 \mathrm{~km}$ from household cluster

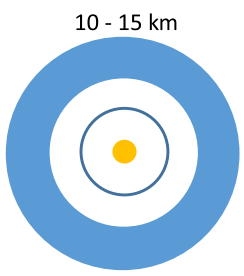

Clinic-level facilities

Health Centers

Hospitals

Note.

The yellow dot in the middle represents a household cluster

Each ring represents a distance buffer (a total of three buffers)

The blue shaded area represents where the health facilities are located

There are a total of 9 main predictor variables

Fig. 1 Main Predictors of the Analysis 
whether or not women were checked before facility discharge and whether or not the newborns were checked before facility discharge were also included in the models. Number of antenatal visits was not included in the models due to potential endogeneity. Types of facilities and their proximity to household clusters could influence decisions regarding antenatal visits. Antenatal visits could also mediate the effects of facilities on PNC use, which is a classic case of endogeneity [21] where it is correlated with the error term when left in the models. Hence, the reported effects of health facilities are total effects, rather than direct effects, which account for the omitted mediated pathways (in the model) through antenatal visits.

Season in which women gave birth was coded as "warm-wet season (November to April)", "winter-dry season (May to August)" or "hot-dry season (September and October)". It was meant to proxy varying road conditions due to seasonal rainfalls. Ownership of TV or a radio was a binary variable meant to proxy potential exposure to health messages in the media. Obtaining money for treatment of any sickness being a big perceived problem was a binary variable meant to proxy financial barriers to accessing care. Women's age at the time of the survey was coded as " $15-24$ ", "25 - 34" and " $35-49$ ". Women's education was coded as having "no education", "primary education" and "secondary education or higher". Employment was a binary variable with " 1 " indicating currently working in either formal or non-formal sectors (including but not limited to agricultural, fishery and sales) and " 0 " otherwise. Household wealth was a rural-specific quintile variable constructed by DHS using principal components analysis [11]. It was coded as "poorest", "poorer", "middle", "richer" or "richest". Number of total births was coded as "1", " $2-3$ " and " 4 or more". Newborn size was subjectively reported by the respondents as either "very large", "larger than average", "average", "smaller than average" or "very small". This variable was meant to proxy potential maternal and/or newborn complications. Newborn sex was coded as "male" or "female". Religion was coded as "Catholic", "Other Christian" or "Muslim, no religion or other unspecified religion". Those with no religion or other religion were less than $1 \%$ and for this reason, they were grouped together with those of Muslim faith, the second smallest group. Region was coded as "Northern", "Central" and "Southern" which are three administrative regions in Malawi. Cesarean section was a binary variable meant to proxy maternal complications. Whether or not mothers received a check before facility discharge and whether or not newborns received a check before facility discharge were both coded as binary variables. These two variables and cesarean section were only included for women who delivered at health facilities.

\section{Analysis}

A series of descriptive analyses were conducted. Then, generalized estimating equations (GEE) were used in STATA version 15.1 for all of the binary outcomes, with each in separate models. Clustering of households was accounted for by specifying the error correlation structure to be "exchangeable" which means that the variance-covariance matrix for each household cluster has an identical structure [22].

In equation form, the GEE models including the aforementioned outcomes, main predictors and covariates can be summed up below. For simplicity, the meaning of each unfamiliar notation is explained in the corresponding subscript.

$$
\begin{aligned}
Y_{\text {outcomes }}= & \alpha_{\text {intercept }}+\beta_{1} \text { Clinics }_{0-5 \mathrm{~km}} \\
& +\beta_{2} \text { Health }_{\text {Centers }} 0-5 \mathrm{~km} \\
& +\beta_{3} \text { Hospitals }_{0-5 \mathrm{~km}}+\beta_{4} \text { Clinics }_{5-10 \mathrm{~km}} \\
& +\beta_{5} \text { Health Centers }_{5-10 \mathrm{~km}} \\
& +\beta_{6} \text { Hospitals }_{5-10 \mathrm{~km}}+\beta_{7} \text { Clinics }_{10-15 \mathrm{~km}} \\
& +\beta_{8} \text { Health Centers }_{10-15 \mathrm{~km}} \\
& +\beta_{9} \text { Hospitals }_{10-15 \mathrm{~km}}+\beta_{X} X_{\text {covariates }} \\
& +\varepsilon_{\text {error term }}
\end{aligned}
$$

The coefficients, denoted by 's, were converted into differential effects in STATA using the "margins" command [23]. Differential effects were derived because they are more intuitive to understand than interpreting odds ratios. Differential effects can be obtained by first calculating the predicted probability of the referent category and the predicted probability of the alternative category and then taking the difference between the two. A general interpretation would be percentage point changes in the probability of the outcome given the alternative condition compared to being in the referent category. More specifically, a full interpretation for $\beta_{1}$, for example, would be the percentage point changes in the average probability of receiving PNC associated with having a clinic-level facility providing PNC within $5 \mathrm{~km}$ compared to not having a clinic-level facility providing PNC within $5 \mathrm{~km}$ (averaged across all household clusters). This is controlling for the distribution of other health facilities providing PNC within $5 \mathrm{~km}$, between $5 \mathrm{~km}$ and $10 \mathrm{~km}$ and between $10 \mathrm{~km}$ and $15 \mathrm{~km}$ as well as aforementioned covariates included in the models. In order to avoid repetition, however, a shortened version of the interpretation is presented in the results section.

Differential effects were only calculated and reported for the main predictors, as they are the focus of the analyses and covariates were carefully selected in order to obtain estimates that are as unbiased as possible for the main predictors. Lastly, all analyses, except those that are only focused on type of health facility and distance were weighted by individual women's sampling probabilities. 


\section{Results}

\section{Background characteristics}

In rural Malawi, a weighted total of about 11,576 women gave birth in the past 5 years preceding the survey (See Table 1). Close to two-fifths of the women were between ages 15 and $24(37.7 \%)$ and more than two-fifths had four or more births (41.4\%). Most women had no formal education (74.8\%) and were currently working in either formal or informal work (67.9\%). In addition, about $66 \%$ of women belonged in the poorest, poorer or middle wealth quintile and $62 \%$ did not own a TV or a radio. Most women were either Catholic or Christian (of other denominations). About $16 \%$ of women were either Muslim, had other unspecified religion or no religion at all. Those with no religion or other religion were less than $1 \%$ (not shown). In terms of perceived barriers in accessing general health care services, over half of the women considered cost of treatment (of any sickness) to be a problem $(57.0 \%)$. Close to $90 \%$ of the women lived in Central and Southern regions of Malawi. For their most recent childbirth, the majority of rural women delivered at a health facility (93.0\%) and only about 5\% had a cesarean section. About half gave birth during the warm and wet season (48.4\%) and reported having an average-sized newborn (49.3\%). Among those who delivered at a health facility, about $46 \%$ reported receiving a maternal health check before discharge and 66\% reported receiving a newborn health check before discharge. The sex of the newborns were close to even.

\section{Availability of health facility types}

In terms of health facility availability, nearly $32 \%$ of rural women had no health facilities within $5 \mathrm{~km}$ of where they lived (See Table 2), with the Central and Northern regions having significantly higher percentages with no facilities (37.2 and 32.5\% respectively). Very few women lived in areas where there were no health facilities within $10 \mathrm{~km}$ of where they lived (2.4\%). However, the Northern region had a significantly higher percentage than the rest of the regions (7.2\%). Within $15 \mathrm{~km}$, nearly all women had a health facility.

Among women living within $5 \mathrm{~km}$ of any clinic-level facility, the percentage of those living within $5 \mathrm{~km}$ of clinic-level facilities that provide PNC services was $25 \%$ (See Table 3). For women living within $5 \mathrm{~km}$ of any health center or hospital, over $90 \%$ were living within $5 \mathrm{~km}$ of health centers and hospitals that provide PNC services. There was a similar pattern for other distance bands as well.

Regarding availability of health facilities that provide PNC (See Table 2), very few women were living in areas where there were clinic-level health facilities providing PNC within $5 \mathrm{~km}$ (5.5\%). In the same distance band (within $5 \mathrm{~km}$ ), the percentage living in areas where there were health centers providing PNC was much higher at around $46 \%$. In the Northern region, a significantly lower percentage of women lived in areas where there were health centers providing $\mathrm{PNC}$ within $5 \mathrm{~km}$ (34.0\%). About 17\% of women had hospitals providing PNC within $5 \mathrm{~km}$ of where they lived. Notably, the percentage having hospitals providing PNC within $5 \mathrm{~km}$ was highest for those living in the Northern region at $27 \%$.

In the distance band between $5 \mathrm{~km}$ and $10 \mathrm{~km}$, about $17 \%$ of women had clinic-level facilities providing PNC. In the Northern region, this percentage was significantly lower at around $8 \%$. With regards to health centers providing $\mathrm{PNC}$ in the same distance band (between $5 \mathrm{~km}$ and $10 \mathrm{~km}$ ), the percentage was much higher at around $70 \%$. However, the Northern region had the lowest percentage yet again (43.5\%). About $24 \%$ of women had hospitals providing PNC in this distance band (between $5 \mathrm{~km}$ and $10 \mathrm{~km})$.

Between $10 \mathrm{~km}$ and $15 \mathrm{~km}$, the percentage having clinic-level facilities providing PNC was about 21\% and the percentage having health centers providing PNC was about $81 \%$. The percentage having hospitals providing PNC was about $35 \%$.

Taken together, nearly $61 \%$ of women had some type of health facility (clinic, health center, or hospital) providing $\mathrm{PNC}$ within $5 \mathrm{~km}$ of where they lived and of women who had no facility providing PNC within $5 \mathrm{~km}$, about $35 \%$ had some type of facility PNC availability between $5 \mathrm{~km}$ and $10 \mathrm{~km}$. This indicates that close to $96 \%$ of women had some degree of access to facility PNC within $10 \mathrm{~km}$ of where they lived. The Central region had the lowest percentage of women having a facility providing PNC within $5 \mathrm{~km}(51.6 \%)$ and the Northern region had the lowest percentage of women having a facility providing PNC within $10 \mathrm{~km}$ (90.4\%).

\section{Place and timing of PNC}

Among rural women who delivered in the past 5 years preceding the survey, about $3 \%$ reported receiving maternal PNC within the first day and about 16\% reported receiving maternal PNC within the first week (See Table 4). For newborns, nearly $3 \%$ had PNC within the first day and about 26\% had PNC within the first week.

Most maternal and newborn PNC was provided at health facilities, 94 and $95 \%$ respectively (See Table 5). For those who delivered at home and received PNC, about 29\% had their maternal PNC and about 16\% had their newborn PNC also at home. Among the same group of women, $71 \%$ had their maternal PNC and $31 \%$ had their newborn PNC within the first $24 \mathrm{~h}$. For those who delivered at health facilities and received PNC, about $10 \%$ had maternal PNC and about $4 \%$ had newborn PNC within the first $24 \mathrm{~h}$. 
Table 1 Background characteristics of rural women who gave birth in the past 5 years preceding the survey in Malawi, MDHS 2015-16

$\frac{\text { Total }}{\mathrm{n}}$

$$
\begin{aligned}
& \text { Age } \\
& \begin{array}{r}
15-24 \\
25-34 \\
35-49
\end{array}
\end{aligned}
$$$$
4368
$$$$
4879
$$$$
2329
$$

Education

None

Primary

8663

2453

Secondary or higher

Employment

Yes

No

7861

3715

Rural Wealth Quintile

Poorest
Poorer
Middle
Richer
Richest

Religion

Catholic
Other Christian

Muslim/other/no religion

Number of births

$\begin{array}{ccc}1 & 2655 & 22.9 \% \\ 2-3 & 4126 & 35.6 \% \\ 4+ & 4795 & 41.4 \% \\ \text { TV/Radio Ownership } & & \\ \text { Yes } & 4370 & 38.1 \% \\ \text { No } & 7111 & 61.9 \%\end{array}$

Cost of treatment (of any sickness) being a perceived problem

$\begin{array}{lll}\text { Yes } & 6602 & 57.0 \% \\ \text { No } & 4974 & 43.0 \% \\ \text { Region of residence } & & \\ \text { Northern } & 1360 & 11.8 \% \\ \text { Central } & 4865 & 42.0 \% \\ \text { Southern } & 5351 & 46.2 \% \\ \text { Place of delivery } & & \\ \text { Home } & 798 & 7.0 \% \\ \text { Health Facility } & 10,599 & 93.0 \% \\ \text { C-section } & & \\ \text { Yes } & 624 & 5.4 \% \\ \text { No } & 10,911 & 94.6 \%\end{array}$

\begin{tabular}{|c|c|c|}
\hline & \multicolumn{2}{|l|}{ Total } \\
\hline & $\mathrm{n}$ & $\%$ \\
\hline \multicolumn{3}{|c|}{ Maternal health check before discharge from the health facility } \\
\hline Yes & 4847 & $45.9 \%$ \\
\hline No & 5711 & $54.1 \%$ \\
\hline \multicolumn{3}{|c|}{ Newborn health check before discharge from the health facility } \\
\hline Yes & 6940 & $65.7 \%$ \\
\hline No & 3618 & $34.3 \%$ \\
\hline \multicolumn{3}{|l|}{ Newborn size } \\
\hline Very large & 1112 & $9.7 \%$ \\
\hline Larger than average & 2831 & $24.7 \%$ \\
\hline Average & 5659 & $49.3 \%$ \\
\hline Smaller than average & 1354 & $11.8 \%$ \\
\hline Very small & 519 & $4.5 \%$ \\
\hline \multicolumn{3}{|l|}{ Newborn sex } \\
\hline Male & 5805 & $50.2 \%$ \\
\hline Female & 5771 & $49.9 \%$ \\
\hline \multicolumn{3}{|c|}{ Season in which women gave birth } \\
\hline Warm-wet & 5604 & $48.4 \%$ \\
\hline Winer-dry & 3733 & $32.3 \%$ \\
\hline Hot-dry & 2239 & $19.3 \%$ \\
\hline
\end{tabular}

Table 1 Background characteristics of rural women who gave birth in the past 5 years preceding the survey in Malawi, MDHS 2015-16 (Continued)

There were 11,576 rural women who gave birth in the past 5 years preceding the survey (weighted)

Columns within a categorical variable sum to $100 \%$

Observations are weighted counts

\section{Interpretation of the reported GEE effects}

All reported effects in the following sections were interpreted as positive or negative associations with maternal/ newborn PNC (averaged across all household clusters) controlling for the existing distribution of health facilities within $5 \mathrm{~km}$, between $5 \mathrm{~km}$ and $10 \mathrm{~km}$ and between $10 \mathrm{~km}$ and $15 \mathrm{~km}$ and also controlling for other covariates aforementioned. The referent group for these effects (of the health facilities) is not having the corresponding type of health facility in the same distance band. For example, the effect of having a health center within $5 \mathrm{~km}$ on maternal/ newborn PNC would be in comparison to not having a health center within $5 \mathrm{~km}$ (averaged across all household clusters), controlling for the distribution of other health facilities within $5 \mathrm{~km}$, between $5 \mathrm{~km}$ and $10 \mathrm{~km}$ and between $10 \mathrm{~km}$ and $15 \mathrm{~km}$ and also controlling for other covariates. It is important to keep in mind that these are population-average estimates.

\section{Effects of health facilities on PNC for women delivering at home}

Among women who delivered at home, having a health center providing $\mathrm{PNC}$ within $5 \mathrm{~km}$ was positively 
Table 2 Availability of health facilities providing PNC within three distance bands of rural household clusters in Malawi, Malawi SPA 2013-14 \& Malawi DHS 2015-16

\begin{tabular}{|c|c|c|c|c|c|c|c|c|}
\hline & \multirow{2}{*}{\multicolumn{2}{|c|}{ Total }} & \multicolumn{6}{|c|}{ Regions } \\
\hline & & & \multicolumn{2}{|c|}{ Northern } & \multicolumn{2}{|c|}{ Central } & \multicolumn{2}{|c|}{ Southern } \\
\hline & $\mathrm{n}$ & $\%$ & $\mathrm{n}$ & $\%$ & $\bar{n}$ & $\%$ & n & $\%$ \\
\hline \multicolumn{9}{|c|}{ Women who have no health facilities } \\
\hline within $5 \mathrm{~km} * * *$ & 6088 & $31.5 \%$ & 1205 & $32.5 \%$ & 2450 & $37.2 \%$ & 2433 & $27.0 \%$ \\
\hline within $10 \mathrm{~km}{ }^{* * *}$ & 463 & $2.4 \%$ & 267 & $7.2 \%$ & 113 & $1.7 \%$ & 83 & $0.9 \%$ \\
\hline within $15 \mathrm{~km}^{* * *}$ & 83 & $0.4 \%$ & 54 & $1.5 \%$ & 0 & $0.0 \%$ & 29 & $0.3 \%$ \\
\hline \multicolumn{9}{|c|}{ Women who have health facilities providing PNC } \\
\hline within $5 \mathrm{~km} * * *$ & 11,719 & $60.7 \%$ & 2289 & $61.8 \%$ & 3395 & $51.6 \%$ & 6035 & $66.9 \%$ \\
\hline $\begin{array}{l}\text { between } 5 \mathrm{~km} \text { and } 10 \mathrm{~km} \\
\text { (but none within } 5 \mathrm{~km})^{* * *}\end{array}$ & 6743 & $34.9 \%$ & 1061 & $28.6 \%$ & 2882 & $43.8 \%$ & 2800 & $31.0 \%$ \\
\hline within $10 \mathrm{~km} * * *$ & 18,462 & $95.6 \%$ & 3350 & $90.4 \%$ & 6277 & $95.4 \%$ & 8835 & $97.9 \%$ \\
\hline \multicolumn{9}{|c|}{ Type of facility providing PNC within $5 \mathrm{~km}$} \\
\hline Clinic-level facilities*** & 1064 & $5.5 \%$ & 179 & $4.8 \%$ & 275 & $4.2 \%$ & 610 & $6.8 \%$ \\
\hline Health centers*** & 8955 & $46.4 \%$ & 1261 & $34.0 \%$ & 2675 & $40.6 \%$ & 5019 & $55.6 \%$ \\
\hline Hospitals*** & 3334 & $17.3 \%$ & 1005 & $27.1 \%$ & 809 & $12.3 \%$ & 1520 & $16.8 \%$ \\
\hline \multicolumn{9}{|c|}{ Type of facility providing PNC between $5 \mathrm{~km}$ and $10 \mathrm{~km}$} \\
\hline Clinic-level facilities*** & 3364 & $17.4 \%$ & 298 & $8.0 \%$ & 786 & $11.9 \%$ & 2280 & $25.3 \%$ \\
\hline Health centers*** & 13,510 & $70.0 \%$ & 1611 & $43.5 \%$ & 4491 & $68.2 \%$ & 7408 & $82.1 \%$ \\
\hline Hospitals*** & 4662 & $24.1 \%$ & 668 & $18.0 \%$ & 1454 & $22.1 \%$ & 2540 & $28.1 \%$ \\
\hline \multicolumn{9}{|c|}{ Type of facility providing PNC between $10 \mathrm{~km}$ and $15 \mathrm{~km}$} \\
\hline Clinic-level facilities*** & 4016 & $20.8 \%$ & 543 & $14.7 \%$ & 859 & $13.1 \%$ & 2614 & $29.0 \%$ \\
\hline Health centers*** & 15,707 & $81.3 \%$ & 2344 & $63.3 \%$ & 5591 & $84.9 \%$ & 7772 & $86.1 \%$ \\
\hline Hospitals*** & 6758 & $35.0 \%$ & 992 & $26.8 \%$ & 2098 & $31.9 \%$ & 3668 & $40.6 \%$ \\
\hline
\end{tabular}

Total number of observations is 19,315

Column percentages were reported corresponding to the category

Observations belonging in different categories of facilities within each buffer are not mutually exclusive (i.e. one observation could be counted multiple times if it has a clinic-level facility, a health center and a hospital all within $5 \mathrm{~km}$ of where it is)

${ }^{* * *} p<0.001$; bivariate chi-square tests were performed (each level of facility vs. regions)

Table 3 Availability of PNC services among health facilities, Malawi DHS 2015-16

\begin{tabular}{lccc}
\hline & Total & \multicolumn{2}{c}{ Facilities providing PNC } \\
\cline { 3 - 4 } & $\mathrm{N}$ & $\mathrm{N}$ & $\%$ \\
\hline Within 5 km of household cluster & & \\
Clinic-level & 4226 & 1064 & $25.2 \%$ \\
Health center & 9536 & 8955 & $93.9 \%$ \\
Hospital & 3357 & 3334 & $99.3 \%$ \\
Between $5 \mathrm{~km}$ and $10 \mathrm{~km}$ of household cluster & \\
Clinic-level & 8200 & 3364 & $41.0 \%$ \\
Health center & 14,193 & 13,510 & $95.2 \%$ \\
Hospital & 4817 & 4662 & $96.8 \%$ \\
Between $10 \mathrm{~km}$ and $15 \mathrm{~km}$ of household cluster & \\
Clinic-level & 10,363 & 4016 & $38.8 \%$ \\
Health center & 16,253 & 15,707 & $96.6 \%$ \\
Hospital & 6813 & 6758 & $99.2 \%$ \\
\hline
\end{tabular}

Total number of observations is 19,315 associated with maternal PNC within the first day and within 7 days (See Table 6). Having a hospital providing PNC farther out (between $5 \mathrm{~km}$ and $10 \mathrm{~km}$ ) was positively associated with maternal PNC within 7 days.

The effects of health facilities on newborn PNC showed slightly different patterns. Having a health center or a

Table 4 Percentages of rural women who gave birth in the past 5 years preceding the survey with maternal/newborn PNC, MDHS 2015-16

\begin{tabular}{lll}
\hline & Total \\
\cline { 2 - 3 } & $\mathrm{n}$ & $\%$ \\
\hline Maternal PNC within 24 $\mathrm{h}$ & 367 & $3.2 \%$ \\
Maternal PNC within the first week & 1877 & $16.2 \%$ \\
Newborn PNC within 24 $\mathrm{h}$ & 340 & $2.9 \%$ \\
Newborn PNC within the first week & 2992 & $25.9 \%$ \\
\hline
\end{tabular}

There were 11,576 rural women who gave birth in the past 5 years preceding the survey (weighted) 
Table 5 Place and timing of maternal/newborn PNC among rural women receiving PNC in Malawi, Malawi DHS 2015-16

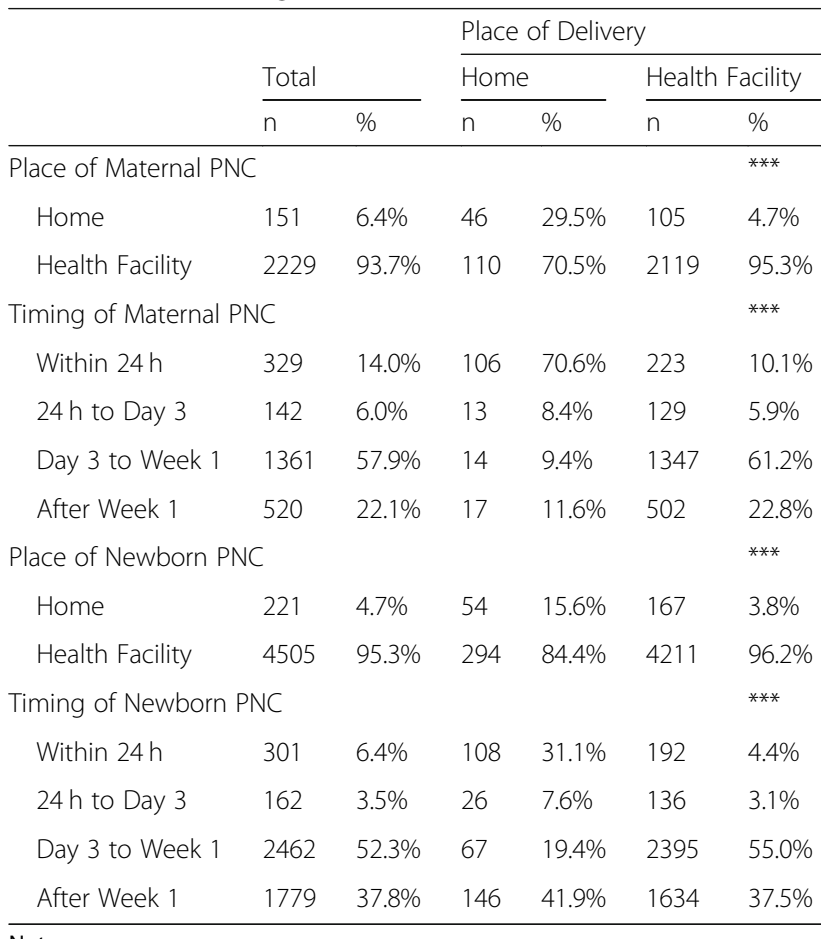

Note.

${ }^{*} p<0.05 * p<<0.01 * * p<0.001$

Total number of women who delivered at the health facility is 10,266 ; Number of observations for maternal outcomes is 10,083; Number of observations for newborn outcomes is 10,029

The outcomes were Maternal PNC between day 1 and day 7 or Newborn PNC between day 1 and day 7 in two separate GEE models

The main predictors (separate binary indicators) in the GEE models were whether or not there was: a clinic-level facility providing PNC within $5 \mathrm{~km}$; a health center providing PNC within $5 \mathrm{~km}$; a hospital providing PNC within 5 $\mathrm{km}$; a clinic-level facility providing PNC between $5 \mathrm{~km}$ and $10 \mathrm{~km}$; a health center providing PNC between $5 \mathrm{~km}$ and $10 \mathrm{~km}$; a hospital providing PNC between $5 \mathrm{~km}$ and $10 \mathrm{~km}$; a clinic-level facility providing PNC between $10 \mathrm{~km}$ and $15 \mathrm{~km}$; a health center providing PNC between $10 \mathrm{~km}$ and $15 \mathrm{~km}$; a hospital providing PNC between $10 \mathrm{~km}$ and $15 \mathrm{~km}$

Covariates included in the GEE models were season in which women gave birth, ownership of TV or radio, whether cost of treatment is a perceived problem, women's age, women's education, women's employment, household wealth, number of births, newborn size, newborn sex, religion, region, cesarean section and whether or not the mother or the newborn (depending on the outcome) was checked before discharge from facility

hospital providing PNC within $5 \mathrm{~km}$ was positively associated with newborn PNC within the first day. There were no other significant facility effects on newborn PNC.

\section{Effects of health facilities on PNC for women delivering at health facilities}

Among women delivering in health facilities, having a health center providing PNC within $5 \mathrm{~km}$ was positively associated with maternal PNC between day 1 and day 7 (See Table 7). Having a health center providing PNC farther out (between $5 \mathrm{~km}$ and $10 \mathrm{~km}$ ) was also positively associated with maternal PNC between day 1 and day 7 . However, having a hospital providing PNC in this distance band (between $5 \mathrm{~km}$ and $10 \mathrm{~km}$ ) was negatively associated with maternal PNC between day 1 and day 7 .

With regards to newborn PNC, having a clinic-level facility providing $\mathrm{PNC}$ within $5 \mathrm{~km}$ was negatively associated with newborn PNC between day 1 and day 7 . Having a clinic-level facility providing PNC farther out (between $5 \mathrm{~km}$ and $10 \mathrm{~km}$ ) was also negatively associated with newborn PNC between day 1 and day 7 but having a health center providing PNC in this distance band (between $5 \mathrm{~km}$ and $10 \mathrm{~km}$ ) was positively associated with newborn PNC between day 1 and day 7. For the effects of the control variables, see Additional file 2 .

\section{Discussion}

This study offered a unique opportunity to examine availability of different types of health facilities around rural household clusters in Malawi and how these facility types at varying distances influence maternal and newborn PNC. This study also highlighted that use of timely maternal and newborn PNC were very low among rural women who gave birth in the past 5 years preceding the survey. Only about 3\% of all delivering mothers and 3\% of newborns received PNC within the first $24 \mathrm{~h}$ of birth. Receipt of PNC within the first week of birth was also low at about $16 \%$ for mothers and $26 \%$ for newborns. This suggests that increasing timely maternal and newborn PNC in general warrants more programmatic focus and attention going forward. The discussion below offers additional insight as to how we can diversify strategies based on women's place of delivery.

\section{Implications for PNC after home delivery}

In terms of women delivering at home, having a health center providing PNC within $5 \mathrm{~km}$ was positively associated with maternal PNC. Having a health center providing PNC within $5 \mathrm{~km}$ was also positively associated with newborn PNC within the first day. The positive effects of health centers may generally be due to their offering of a wider range of basic client services and delivery-related services compared to other lower-level facilities [18] and therefore, they might have higher recognition to women living close by. Clinic-level facilities, on the other hand, may not have the same level of recognition or familiarity to women because many of these facilities do not provide the range of maternity services that the health centers can [18].

Based on these findings, a few strategies can be considered to increase utilization of PNC after home delivery. First, training health providers based in clinic-level facilities to provide both maternal and newborn PNC could lead to more sites where PNC is available. Among women who lived within $5 \mathrm{~km}$ of a clinic-level facility, only about $25 \%$ had clinic-level facilities providing PNC. Second, and more importantly, emphasizing quality by ensuring that the recommended content of PNC is 
Table 6 The effects of different types and proximities of health facilities on maternal/newborn PNC among rural women who gave birth at home in Malawi, MDHS 2015-16

\begin{tabular}{|c|c|c|c|c|c|c|c|c|}
\hline & \multicolumn{4}{|c|}{ Maternal } & \multicolumn{4}{|c|}{ Newborn } \\
\hline & \multicolumn{2}{|c|}{ PNC within 1 day } & \multicolumn{2}{|c|}{ PNC within 7 days } & \multicolumn{2}{|c|}{ PNC within 1 day } & \multicolumn{2}{|c|}{ PNC within 7 days } \\
\hline & $\overline{\mathrm{DE}}$ & {$[95 \% \mathrm{Cl}]$} & $\overline{\mathrm{DE}}$ & {$[95 \% \mathrm{Cl}]$} & $\overline{\mathrm{DE}}$ & {$[95 \% \mathrm{Cl}]$} & $\overline{\mathrm{DE}}$ & {$[95 \% \mathrm{Cl}]$} \\
\hline \multicolumn{9}{|c|}{ Type and Proximity of Health Facilities } \\
\hline \multicolumn{9}{|c|}{ Within $5 \mathrm{~km}$ of household cluster } \\
\hline \multicolumn{9}{|l|}{ Clinic-level } \\
\hline No facility (ref) & - & - & - & - & - & - & - & - \\
\hline Facility & 0.046 & {$[-0.091,0.184]$} & 0.124 & {$[-0.012,0.259]$} & -0.035 & {$[-0.179,0.108]$} & 0.042 & {$[-0.137,0.221]$} \\
\hline \multicolumn{9}{|l|}{ Health center } \\
\hline No facility (ref) & - & - & - & - & - & - & - & - \\
\hline Facility & $0.079^{* *}$ & {$[0.020,0.138]$} & $0.073^{*}$ & {$[0.012,0.135]$} & $0.068^{*}$ & {$[0.003,0.132]$} & 0.063 & {$[-0.020,0.146]$} \\
\hline \multicolumn{9}{|l|}{ Hospital } \\
\hline No facility (ref) & - & - & - & - & - & - & - & - \\
\hline Facility & 0.028 & {$[-0.053,0.108]$} & -0.002 & {$[-0.087,0.082]$} & $0.137^{*}$ & {$[0.012,0.263]$} & 0.117 & {$[-0.053,0.287]$} \\
\hline \multicolumn{9}{|c|}{ Between $5 \mathrm{~km}$ and $10 \mathrm{~km}$ of household cluster } \\
\hline \multicolumn{9}{|l|}{ Clinic-level } \\
\hline No facility (ref) & - & - & - & - & - & - & - & - \\
\hline Facility & -0.032 & {$[-0.110,0.046]$} & -0.003 & {$[-0.076,0.069]$} & 0.030 & {$[-0.034,0.094]$} & 0.037 & {$[-0.045,0.118]$} \\
\hline \multicolumn{9}{|l|}{ Health center } \\
\hline No facility (ref) & - & - & - & - & - & - & - & - \\
\hline Facility & 0.039 & {$[-0.028,0.105]$} & 0.028 & {$[-0.037,0.092]$} & 0.028 & {$[-0.043,0.099]$} & 0.041 & {$[-0.039,0.121]$} \\
\hline \multicolumn{9}{|l|}{ Hospital } \\
\hline No facility (ref) & - & - & - & - & - & - & - & - \\
\hline Facility & 0.062 & {$[-0.0004,0.125]$} & $0.073^{*}$ & {$[0.012,0.134]$} & 0.030 & {$[-0.029,0.089]$} & 0.066 & {$[-0.015,0.148]$} \\
\hline \multicolumn{9}{|c|}{ Between $10 \mathrm{~km}$ and $15 \mathrm{~km}$ of household cluster } \\
\hline \multicolumn{9}{|l|}{ Clinic-level } \\
\hline No facility (ref) & - & - & - & - & - & - & - & - \\
\hline Facility & -0.062 & {$[-0.134,0.010]$} & -0.074 & {$[-0.150,0.003]$} & -0.009 & {$[-0.077,0.060]$} & 0.006 & {$[-0.082,0.093]$} \\
\hline \multicolumn{9}{|l|}{ Health center } \\
\hline No facility (ref) & - & - & - & - & - & - & - & - \\
\hline Facility & -0.012 & {$[-0.093,0.070]$} & -0.035 & {$[-0.119,0.049]$} & 0.050 & {$[-0.038,0.138]$} & -0.020 & {$[-0.123,0.082]$} \\
\hline \multicolumn{9}{|l|}{ Hospital } \\
\hline No facility (ref) & - & - & - & - & - & - & - & - \\
\hline Facility & 0.027 & {$[-0.034,0.087]$} & 0.025 & {$[-0.042,0.092]$} & -0.000 & {$[-0.059,0.059]$} & 0.009 & {$[-0.072,0.089]$} \\
\hline
\end{tabular}

Total number of women who delivered at home is 691 ; Number of observations for maternal outcomes is 665 ; Number of observations for newborn outcomes is 664

The outcomes were Maternal PNC within 1 day, Maternal PNC within 7 days, Newborn PNC within 1 day or Newborn PNC within 7 days in four separate GEE models

The main predictors (separate binary indicators) in the GEE models were whether or not there was: a clinic-level facility providing PNC within 5 km; a health center providing PNC within $5 \mathrm{~km}$; a hospital providing PNC within $5 \mathrm{~km}$; a clinic-level facility providing PNC between $5 \mathrm{~km}$ and $10 \mathrm{~km}$; a health center providing PNC between $5 \mathrm{~km}$ and $10 \mathrm{~km}$; a hospital providing PNC between $5 \mathrm{~km}$ and $10 \mathrm{~km}$; a clinic-level facility providing PNC between $10 \mathrm{~km}$ and $15 \mathrm{~km}$; a health center providing PNC between $10 \mathrm{~km}$ and $15 \mathrm{~km}$; a hospital providing PNC between $10 \mathrm{~km}$ and $15 \mathrm{~km}$

Covariates included in the GEE models were season in which women gave birth, ownership of TV or radio, whether cost of treatment is a perceived problem, women's age, women's education, women's employment, household wealth, number of births, newborn size, newborn sex, religion and region ${ }^{*} p<0.05{ }^{* *} p<0.01$

provided in these lower-level facilities can be an effective intervention strategy. This is because clinic-level facilities already providing PNC were not shown to be positively associated with maternal or newborn PNC. It would be important to support these lower-level facilities to provide quality PNC and to inform local 
Table 7 The effects of different types and proximities of health facilities on maternal/newborn PNC among rural women who gave birth at health facilities in Malawi, MDHS 2015-16

\begin{tabular}{|c|c|c|c|c|}
\hline & \multicolumn{2}{|l|}{ Maternal } & \multicolumn{2}{|l|}{ Newborn } \\
\hline & \multicolumn{2}{|c|}{ PNC between Day 1 and Day 7} & \multicolumn{2}{|c|}{ PNC between Day 1 and Day 7} \\
\hline & $\mathrm{DE}$ & {$[95 \% \mathrm{Cl}]$} & $\mathrm{DE}$ & {$[95 \% \mathrm{Cl}]$} \\
\hline \multicolumn{5}{|c|}{ Type and Proximity of Health Facilities } \\
\hline \multicolumn{5}{|c|}{ Within $5 \mathrm{~km}$ of household cluster } \\
\hline \multicolumn{5}{|l|}{ Clinic-level } \\
\hline No facility (ref) & - & - & - & - \\
\hline Facility & -0.023 & {$[-0.077,0.032]$} & $-0.134^{* *}$ & {$[-0.210,-0.057]$} \\
\hline \multicolumn{5}{|l|}{ Health center } \\
\hline No facility (ref) & - & - & - & - \\
\hline Facility & $0.032^{*}$ & {$[0.004,0.059]$} & 0.009 & {$[-0.023,0.041]$} \\
\hline \multicolumn{5}{|l|}{ Hospital } \\
\hline No facility (ref) & - & - & - & - \\
\hline Facility & 0.034 & {$[-0.005,0.074]$} & 0.020 & {$[-0.024,0.065]$} \\
\hline \multicolumn{5}{|c|}{ Between $5 \mathrm{~km}$ and $10 \mathrm{~km}$ of household cluster } \\
\hline \multicolumn{5}{|l|}{ Clinic-level } \\
\hline No facility (ref) & - & - & - & - \\
\hline Facility & 0.002 & {$[-0.031,0.034]$} & $-0.047^{*}$ & {$[-0.090,-0.004]$} \\
\hline \multicolumn{5}{|l|}{ Health center } \\
\hline No facility (ref) & - & - & - & - \\
\hline Facility & $0.044^{* *}$ & {$[0.014,0.074]$} & $0.069^{* * *}$ & {$[0.034,0.105]$} \\
\hline \multicolumn{5}{|l|}{ Hospital } \\
\hline No facility (ref) & - & - & - & - \\
\hline Facility & $-0.042^{* *}$ & $\begin{array}{l}{[-0.072} \\
-0.011]\end{array}$ & -0.010 & {$[-0.043,0.023]$} \\
\hline \multicolumn{5}{|c|}{ Between $10 \mathrm{~km}$ and $15 \mathrm{~km}$ of household cluster } \\
\hline \multicolumn{5}{|l|}{ Clinic-level } \\
\hline No facility (ref) & - & - & - & - \\
\hline Facility & -0.004 & {$[-0.036,0.028]$} & 0.030 & {$[-0.010,0.070]$} \\
\hline \multicolumn{5}{|l|}{ Health center } \\
\hline No facility (ref) & - & - & - & - \\
\hline Facility & 0.007 & {$[-0.027,0.041]$} & 0.030 & {$[-0.012,0.072]$} \\
\hline \multicolumn{5}{|l|}{ Hospital } \\
\hline No facility (ref) & - & - & - & - \\
\hline Facility & -0.002 & {$[-0.031,0.027]$} & -0.013 & {$[-0.045,0.019]$} \\
\hline
\end{tabular}

Total number of women who delivered at the health facility is 10,266; Number of observations for maternal outcomes is 10,083; Number of observations for newborn outcomes is 10,029

The outcomes were Maternal PNC between day 1 and day 7 or Newborn PNC between day 1 and day 7 in two separate GEE models

The main predictors (separate binary indicators) in the GEE models were whether or not there was: a clinic-level facility providing PNC within 5 km; a health center providing PNC within $5 \mathrm{~km}$; a hospital providing PNC within $5 \mathrm{~km}$; a clinic-level facility providing PNC between $5 \mathrm{~km}$ and $10 \mathrm{~km}$; a health center providing PNC between $5 \mathrm{~km}$ and $10 \mathrm{~km}$; a hospital providing PNC between $5 \mathrm{~km}$ and $10 \mathrm{~km}$; a clinic-level facility providing PNC between $10 \mathrm{~km}$ and $15 \mathrm{~km}$; a health center providing PNC between $10 \mathrm{~km}$ and $15 \mathrm{~km}$; a hospital providing PNC between $10 \mathrm{~km}$ and $15 \mathrm{~km}$

Covariates included in the GEE models were season in which women gave birth, ownership of TV or radio, whether cost of treatment is a perceived problem, women's age, women's education, women's employment, household wealth, number of births, newborn size, newborn sex, religion, region, cesarean section and whether or not the mother or the newborn (depending on the outcome) was checked before discharge from facility

${ }^{*} p<0.05{ }^{* *} p<0.01{ }^{* * *} p<0.001$ 
communities of such changes in order to encourage use of these existing resources. Third, community health workers could also receive regular training and work closely with clinic-level facilities and health centers to provide PNC services in women's homes.

However, lack of provider training may not be the only issue. A study specifically looking at provision of PNC and uptake in four African countries reported that structures of organizational support and a system of accountability for health workers were not properly in place in Malawi (one of the four countries), leaving workers demotivated to deliver quality PNC [24]. Another study looking at health workers' perspectives on worker retention and motivation in Malawi also found that major demotivating factors for health workers were generally low salary, unclear job descriptions, unequal opportunities for training, lack of an appropriate performance appraisal system and lack of supervision and feedback from the management [25]. These general issues would also be a hindrance to health workers providing quality PNC as well. Hence, coupled with provider training, there may need to be a wider health sector reform at the district management and health facility levels to establish a working accountability, supervision and feedback mechanism for providing quality PNC $[24,26]$.

Fourth, raising community-awareness about the importance of timely PNC and the utility of clinic-level facilities for preventative PNC may also increase demand for service utilization, as there already seems to be high availability of health facilities providing PNC both within $5 \mathrm{~km}$ and within $10 \mathrm{~km}$ and also high acceptability of health facilities. In 2015 and 2016, 91\% of deliveries occurred in a facility setting in Malawi [11]. Hence, given the general availability and acceptability of health facilities, knowing what is offered at clinic-level facilities and health centers can be an important driver of service use. However, it would be important to couple this with quality improvement for PNC services so that women have assurance that they will receive quality preventative PNC in the lower-level facilities. One of the primary roles of clinic-level facilities is to provide preventative health services [27].

There is some evidence from a review study of demandside interventions for maternal care that community-based mobilizations where trained facilitators led various forms of discussion groups to enhance knowledge and awareness of health problems, resulted in increased utilization of facilitybased maternal care [28]. This review study only considered antenatal visits, facility-based delivery and delivery with skilled birth attendants as utilization outcomes [28]. Nonetheless, it showed potential that community-based mobilization interventions can increase use of facilitybased maternal services [28].
Between $5 \mathrm{~km}$ and $10 \mathrm{~km}$, having a hospital providing PNC, compared to not having one, was positively associated with maternal PNC. This indicates that at this distance, women delivering at homes preferred the hospital for maternal PNC visits. As mentioned previously, if clinics and health centers closer by (within $5 \mathrm{~km}$ ) provide adequate PNC to communities, high patient load and burden of care could be shifted away from hospitals both near (within $5 \mathrm{~km}$ ) and far (between $5 \mathrm{~km}$ and $10 \mathrm{~km}$ ).

Strengthening the capacity of lower-level facilities is also beneficial for home PNC visits. This is because facilities will be able to provide more support and training to affiliated community attendants for home outreach and referrals. An evaluation of context-specific interventions designed to improve PNC in Africa found that community health workers can effectively operate as a bridge between women and the formal health sector [24]. Facility-initiated interventions such as training, supervision and other incentive structures were found to strengthen the professional connection between the health facilities and the community health workers and also increase their motivations as well [24]. Community health workers with stronger links to the health facilities are able to identify referral cases during home visits and encourage more women to seek facility-based care [24].

\section{Implications for PNC after facility delivery}

For women delivering at health facilities, a general intervention strategy should involve a streamlined referral system in which all women delivering at the facility are either visited by a health provider while in the facility or encouraged to visit the postnatal ward within the same facility site before going home for the first time after delivery. For women who cannot follow this suggestion, health providers should refer them to health facilities providing PNC near their residences and consider a home visit strategy. As with the proposed intervention strategies for women delivering at home, supporting clinic-level facilities as well as health centers to provide high quality PNC will help women delivering at health facilities to follow up on their providers' referrals since only health centers were found positively associated with PNC visits. Along with the referrals, the literature suggests that providers should also be mindful about their attitude in giving a thorough explanation of the purpose and the benefits of care to women in general $[29,30]$ and when providing maternal health services [31, 32]. In Malawi, a commonly cited reason for not wanting to receive care at the health facilities is providers' lack of explanation and poor attitude [29-32]. Although asking health providers to be more "mindful" about their demeanor seems like a trivial task, it should be done considering the challenges providers in Malawi face $[25,33]$. The Malawian health system struggles with severe understaffing $[25,33]$ and inappropriate skill mix of health 
providers, especially in the delivery ward [33]. As a result, health providers often report having physical, psychological and emotional stress [33]. Adding another task, albeit simple, may not be effective with already over-burdened staff $[25,33]$ if it is not complemented with appropriate organizational and sector-wide reforms boosting health worker motivations to provide quality care [24, 26, 34, 35]. As discussed before, key determinants of health worker motivation include organizational support, accountability, feedback, supervision and incentive structures [24, 26, 34, 35].

Taken together, the proposed strategies have the potential not only to be useful for encouraging timely PNC within the first day but also making it easier for subsequent postnatal visits which are recommended three more times: on day 3 , between the first and second week and at 6 weeks [36]. Importantly, these strategies are also consistent with the Malawi health sector strategic plan for 2017 to 2022 which was published in April of 2017 [27]. This 5-year strategic plan issued by the Malawian Ministry of Health outlines that the Malawian government intends to "increase equitable access to and quality of health care services" in the provision of the "Essential Health Package" [27]. The "Essential Health Package" encompasses a wide range of important health services including treatment of postpartum hemorrhage [27]. However, preventative PNC was not clearly delineated in the "Essential Health Package". Nevertheless, it is still considered a key component of the essential deliveryrelated services [7]. A recommendation would be to have both maternal and newborn PNC clearly listed as essential services in the package. Other relevant objectives of the Malawi health sector strategic plan II are improving the quality of training and performance for health workers and promoting healthy behaviors through community education [27].

\section{Availability of health facilities providing PNC}

Close to two-thirds of rural women had a health facility providing PNC within $5 \mathrm{~km}$ of where they lived with some regional variation. In the larger $10 \mathrm{~km}$ distance band, almost all rural women had health facilities providing PNC. In addition, facilities located beyond $10 \mathrm{~km}$, regardless of type, were not significant in predicting maternal or newborn PNC. Together, these findings suggest that investing in the construction of new clinics or health centers merely to increase availability around rural communities may be a redundant effort, having little to no effect on encouraging higher utilization of PNC. Instead, resources could be directed towards raising community awareness about the importance of timely PNC, supporting quality improvement initiatives for lower-level facilities, ensuring convenient means of transportation and lowering costs of getting to the facilities and receiving care. Among rural Malawian women, only about $4 \%$ possessed either motorcycles, scooters, cars or trucks in their households for transportation [11]. About $42 \%$ listed bicycles as means of transportation [11]. In addition, over half of the women in this study responded that cost of treatment (of any sickness) was a perceived barrier to seeking care.

\section{Possible explanations for negative health facility effects}

There were three statistically significant negative effects of health facilities for women who delivered at health facilities. Although the exact reasons are unknown, this could perhaps be due to several factors: (1) the selection effect of clinic-level facilities being placed in areas where health outcomes are generally poorer and utilization of health services including PNC are low. Hence, their presence is associated with lower PNC use; (2) women having little to no awareness that PNC services are offered, especially in clinic-level facilities; (3) women having low confidence that clinic-level facilities can provide quality PNC; (4) women not being able to follow through with recommended PNC after discharge for their newborns; or (5) women not being convinced that seeking additional PNC is necessary after discharge.

\section{Limitations}

There are a few limitations in the study. First, women who may have been at very high risk and died are not represented in the sample. This could potentially lead to a misrepresentation of the coverage of PNC use. Second, due to missing information, some facilities were not matched in the process of merging facility locations with the provider interviews to determine whether facilities provided PNC service. However, the number of unmatched facilities was small at around 3\%. Third, the design effect of stratification was not accounted for in the analyses. Women's sampling weights and clustering were applied to individual cases however. For comparison, sensitivity analyses were conducted taking into account the full design effect (stratification, clustering and women's sampling weights) with logistic regression and the results were nearly identical to the main results presented in this study (see Additional file 3). The magnitude of the effects were very similar and the signs and the significance of the effects were exactly the same. Fourth, GPS coordinates of the household clusters have been displaced at a random angle and a random distance from their original locations. Although using buffers with reasonable distances to link facilities and household clusters is expected to somewhat account for the displacement [17], it introduces "noise" in the analysis nonetheless. However, even in the presence of such "noise", knowing the exact locations and characteristics of all operational health facilities (based on 2013 data) in 
Malawi allowed the unique opportunity to investigate the study's research questions.

\section{Conclusions}

The main findings of the study offer important insights for future policy considerations in the context of rural Malawi and comparable regions in sub-Saharan Africa at large. Clinic-level facilities and health centers that currently do not provide $\mathrm{PNC}$ should be supported to provide quality PNC to women and newborns. Quality improvement strategies can be considered for lowerlevel facilities that already provide PNC. Women who deliver at health facilities should receive their first PNC visit (for both the mother and the newborn) before they leave the facility as a standard of practice. Health providers should also mindfully explain the importance of timely PNC and refer women to facilities providing PNC near their residences for further visits. Home visits can also be promoted to reach both women who delivered in facilities as well as the smaller number of women who did not. Lastly, allocating resources to the construction of new facilities does not seem to be a good strategy for increasing utilization of PNC. Instead, more effective strategies are: (1) training providers to be able to perform quality PNC at all facilities; (2) establishing a working system of support, accountability, feedback, supervision and incentives at the organizational and district levels to mitigate issues of staff frustrations and lack of motivation; and (3) increasing community awareness about the importance of seeking timely PNC and about the utility of lower-level facilities for receiving preventative $\mathrm{PNC}$.

\section{Supplementary information}

Supplementary information accompanies this paper at https://doi.org/10. 1186/s12884-019-2534-x.

Additional file 1. The effects of different types and proximities of health facilities on maternal/newborn PNC within 1 day among rural women who gave birth at health facilities in Malawi, Malawi DHS 2015-16.

Additional file 2. Stata log output for the covariate effects.

Additional file 3. Stata log output for sensitivity analyses with the full design effect.

\section{Abbreviations}

GEE: Generalized estimating equations; MDHS: Malawi Demographic and Health Survey; MSPA: Malawi Service Provision Assessment; PNC: Postnatal care; SDG: Sustainable Development Goal; WHO: World Health Organization

\section{Acknowledgements}

We thank Dr. Kirsten Kainz (UNC School of Social Work) and Dr. Guang Guo (UNC Department of Sociology) for providing insightful comments on the paper.

\section{Authors' contributions}

ETK led the study design, data management, data analysis and writing and revising of the manuscript. KS and ISS advised on the development of the paper and methods. KS and ISS also read and provided substantial comments on each version of the paper. GA advised on the methods and provided helpful comments on the paper. WW contributed to the discussion of the paper. All authors read and approved the final manuscript.

\section{Funding}

This study was carried out with support provided by the United States Agency for International Development (USAID) through MEASURE Evaluation (cooperative agreement AID-OAA-L-14-00004). The views expressed are not necessarily those of USAID or the United States government.

\section{Availability of data and materials}

The datasets analyzed during the current study are available upon request on the DHS Program website. We encourage interested researchers to request permission to download these datasets directly from the DHS Program. The conditions of use for DHS datasets do not allow us to share study datasets with other researchers without DHS Program's written consent. Please see the following website for details on the terms of use: https://dhsprogram.com/data/terms-of-use.cfm.

\section{Ethics approval and consent to participate}

The authors of this study did not collect information directly from the respondents. Since there was no contact with the respondents, the authors did not collect informed consent themselves. The data were collected and managed by the DHS Program and later distributed to researchers obtaining permission to use the datasets. In addition, this study (secondary data analysis) was reviewed and exempted from needing ethics approval by the Institutional Review Board at the University of North Carolina at Chapel Hill.

\section{Consent for publication}

Not applicable.

\section{Competing interests}

The authors declare that they have no competing interests.

\section{Author details}

'Department of Maternal and Child Health, Gillings School of Global Public Health, University of North Carolina at Chapel Hill, Chapel Hill, NC, USA. ${ }^{2}$ MEASURE Evaluation/Carolina Population Center, University of North Carolina at Chapel Hill, Chapel Hill, NC, USA. ${ }^{3}$ Carolina Population Center, University of North Carolina at Chapel Hill, Chapel Hill, NC, USA. ${ }^{4}$ Department of International Health (Health Systems Program), Johns Hopkins Bloomberg School of Public Health, Baltimore, MD, USA.

Received: 2 April 2019 Accepted: 24 September 2019

Published online: 17 December 2019

\section{References}

1. United Nations. Sustainable Development Goals - 17 goals to transform our world: United Nations; 2015. Available from: http://www.un.org/ sustainabledevelopment/health/. [cited 2017 May 4]

2. WHO, UNICEF, UNFPA, World Bank Group, United Nations Population Division Maternal Mortality Estimation Inter-Agency Group. Maternal mortality in 1990-2015 [Internet]: World Health Organization. Available from: http://www.who.int/gho/maternal_health/countries/mwi.pdf. [cited 2017 Dec 7]

3. WHO, UNICEF, UNFPA, World Bank Group, United Nations Population Division. Trends in Maternal Mortality: 1990 to 2015. Geneva: World Health Organization; 2015.

4. UNICEF, World Health Organization, World Bank Group, United Nations. Levels and Trends in Child Mortality Report 2015. 2015.

5. International Monetary Fund. Malawi Economic Development Document IMF Country Report 17(184). 2017.

6. Organisation for Economic Co-operation and Development (OECD). DEVEL OPMENT AID AT A GLANCE: STATISTICS BY REGION: 2. AFRICA [Internet]. 2018. Available from: http://www.oecd.org/dac/financingsustainable-development/

7. The Partnership for Maternal $\mathrm{N}$ and $\mathrm{CH}$. Opportunities for Africa's newborns: Practical data, policy and programmatic support for newborn care in Africa [Internet]: WHO. World Health Organization; 2006. Available from: http://www.who.int/pmnch/media/publications/ africanewborns/en/. [cited 2017 Dec 7] 
8. World Health Organization. WHO recommendations on postnatal care of the mother and newborn. 2013.

9. Singh K, Brodish P, Haney E. Postnatal care by provider type and neonatal death in sub-Saharan Africa: a multilevel analysis. BMC Public Health. 2014;14:941.

10. Langlois ÉV, Miszkurka M, Zunzunegui MV, Ghaffar A, Ziegler D, Karp I. Inequities in postnatal care in low-and middle-income countries: a systematic review and meta-analysis. Bull World Health Organ. 2015; 93:259-70.

11. National Statistical Office (NSO) [Malawi], ICF International. Malawi Demographic and Health Survey 2015-16 [Internet]. Zomba, Malawi, and Rockville, Maryland, USA; 2017. Available from: http://dhsprogram.com/ pubs/pdf/FR319/FR319.pdf. Accessed 5 Mar 2019.

12. Strasser R. Rural health around the world: challenges and solutions. Fam Pract. 2003:20(4):457-63.

13. Cronin CJ, Guilkey DK, Speizer IS. The individual's choice of facility for maternal health and family planning services in a dense urban environment: the case of Senegal. SSRN [Internet]. 2016. Available from: https://doi.org/10.2139/ssrn.2848295. Accessed 11 Dec 2017.

14. Strasser R, Kam SM, Regalado SM. Rural Health Care Access and Policy in Developing Countries. Annu Rev Public Health. 2016:37:395-412.

15. World Health Organization. Achieving the health-related MDGs. It takes a workforce! [Internet]. Health workforce: World Health Organization; 2010. Available from: http://www.who.int/hrh/workforce_mdgs/en/ [cited 2018 Sept 7]

16. Burgert CR, Colston J, Roy T, Zachary B. Geographic Displacement Procedure and Georeferenced Data Release Policy for the Demographic and Health Surveys. Calverton: ICF International; 2013.

17. Burgert CR, Prosnitz D. Linking DHS Household and SPA Facility Surveys: Data Considerations and Geospatial Methods [Internet]. Rockville, Maryland, USA; 2014. Available from: https://dhsprogram.com/pubs/pdf/SAR10/SAR10. pdf. Accessed 5 Mar 2019.

18. Ministry of Health (MoH) [Malawi], ICF International. Malawi Service Provision Assessment (MSPA) 2013-14. Lilongwe, Malawi, and Rockville, Maryland, USA. 2014

19. Skiles MP, Burgert CR, Curtis SL, Spencer J. Geographically linking population and facility surveys: methodological considerations. Popul Health Metrics. 2013:11:14.

20. The DHS Program - Quality information to plan, monitor and improve population, health, and nutrition programs [Internet]. Available from: https:// dhsprogram.com/. [cited 2017 Dec 7].

21. Gunasekara Fl, Carter K, Blakely T. Glossary for econometrics and epidemiology. J Epidemiol Community Health. 2008;62(10):858-61.

22. Hubbard AE, Ahern J, Fleischer NL, Van Der Laan M, Satariano SA, Jewell N, et al. To GEE or Not to GEE: Comparing Population Average and Mixed Models for Estimating the Associations Between Neighborhood Risk Factors and Health. Epidemiology. 2010;21(4):467-74.

23. STATA. Margins documentation [Internet]. stata.com. Available from: https:// www.stata.com/manuals13/rmargins.pdf. [cited 2018 June 22]

24. Djellouli N, Mann S, Nambiar B, Meireles P, Miranda D, Barros H, et al. Improving postpartum care delivery and uptake by implementing contextspecific interventions in four countries in Africa: a realist evaluation of the Missed Opportunities in Maternal and Infant Health (MOMI) project. BMJ Glob Health. 2017;2:e000408.

25. Manafa O, McAuliffe E, Maseko F, Bowie C, MacLachlan M, Normand C Retention of health workers in Malawi: perspectives of health workers and district management. Hum Resour Health. 2009;7:65.

26. Rowe AK, De Savigny D, Lanata CF, Victora CG. How can we achieve and maintain high-quality performance of health workers in low-resource settings? Lancet. 2005;366:1026-35.

27. Malawi Ministry of Health. Health Sector Strategic Plan II (2017-2022). Government of the Republic of Malawi. 2017.

28. Hurst TE, Semrau K, Patna M, Gawande A, Hirschhorn LR. Demand-side interventions for maternal care: Evidence of more use, not better outcomes. BMC Pregnancy Childbirth. 2015;15:297.

29. Munthali AC, Mannan H, MacLachlan M, Swartz L, Makupe CM, Chilimampunga C. Non-use of Formal Health Services in Malawi: Perceptions from Non-users. Malawi Med J. 2014;26(4):126-32.

30. Abiiro GA, Mbera GB, De Allegri M. Gaps in universal health coverage in Malawi: A qualitative study in rural communities. BMC Health Serv Res. 2014;14:234.
31. Kambala C, Morse T, Masangwi S, Mitunda P. Barriers to maternal health service use in Chikhwawa, Southern Malawi. Malawi Med J. 2011;23(1):1-5.

32. Kumbani LC, Chirwa E, Malata A, Odland JØ, Bjune G. Do Malawian women critically assess the quality of care? A qualitative study on women's perceptions of perinatal care at a district hospital in Malawi. Reprod Health. 2012;9:30.

33. Bradley S, Kamwendo F, Chipeta E, Chimwaza W, De Pinho H, McAuliffe E. Too few staff, too many patients: a qualitative study of the impact on obstetric care providers and on quality of care in Malawi. BMC Pregnancy Childbirth. 2015;15:65.

34. Nambiar B, Hargreaves DS, Morroni C, Heys M, Crowe S, Pagel C, et al. Improving health-care quality in resource-poor settings. Bull World Health Organ. 2017;95:76-8.

35. Franco LM, Bennett $S$, Kanfer R. Health sector reform and public sector health worker motivation: a conceptual framework. Soc Sci Med. 2002;54:1255-66.

36. World Health Organization. Postnatal Care for Mothers and Newborns: Highlights from the World Health Organization 2013 Guidelines [Internet]. Maternal and Child Survival Program. 2015. Available from: http://www.who. int/maternal_child_adolescent/publications/WHO-MCA-PNC-2014-Briefer_A4 pdf. [cited 2017 Dec 7].

\section{Publisher's Note}

Springer Nature remains neutral with regard to jurisdictional claims in published maps and institutional affiliations.
Ready to submit your research? Choose BMC and benefit from:

- fast, convenient online submission

- thorough peer review by experienced researchers in your field

- rapid publication on acceptance

- support for research data, including large and complex data types

- gold Open Access which fosters wider collaboration and increased citations

- maximum visibility for your research: over $100 \mathrm{M}$ website views per year

At BMC, research is always in progress.

Learn more biomedcentral.com/submissions 